\title{
Defining adherence to therapeutic exercise for musculoskeletal pain: A systematic review.
}

\author{
D Bailey ${ }^{1}$, MA Holden ${ }^{1}$, NE Foster ${ }^{1}$, JG Quicke ${ }^{1}$, KL Haywood ${ }^{2}$, A Bishop ${ }^{1}$ \\ 1. Research Institute for Primary Care and Health Sciences, Keele University. 2. Warwick Research in Nursing, \\ Warwick Medical School, Warwick University.
}

Corresponding author: Daniel Bailey, d.bailey2@keele.ac.uk

\section{Funding:}

D. Bailey is supported for this work through a Keele University, Research Institute for Primary Care and Health Sciences, ACORN PhD Studentship.

N.E Foster and A. Bishop were supported through a National Institute for Health Research (NIHR) Research Professorship awarded to N.E. Foster (NIHR-RP-011-015). Professor N.E. Foster is a NIHR Senior Investigator.

J.G. Quicke is supported by a NIHR Academic Clinical Lectureship in Physiotherapy, awarded as part of Professor C. Mallen's NIHR Research Professorship (NIHR-RP-2014-026).

K.L. Haywood is supported by Warwick Research in Nursing, Warwick Medical School, Warwick University.

The views expressed in this publication are those of the authors and not necessarily those of the NHS, the NIHR or the Department of Health.

\section{Disclosures or conflicts of interest: None}

Word count: 4305 including headings

Keywords: Exercise, adherence, compliance, musculoskeletal pain, definition.

Author contributions - DB, NF, MH \& AB contributed to the design of the review. DB executed the search strategy with input from NF, MH \& AB. DB, NF, AB \& JQ assessed studies for inclusion and extracted data from each of the included studies. DB analysed the data and developed the first draft. All authors interpreted the data, contributed to the critical revision of the manuscript and approved the final version.

Acknowledgements: None 


\section{ABSTRACT (248 words)}

Objective: To establish the meaning of the term 'adherence' (including conceptual and measurement definitions) in the context of therapeutic exercise (TE) for musculoskeletal (MSK) pain.

Design: Systematic review using a search strategy including terms for: adherence, TE and MSK pain. Identified studies were independently screened for inclusion by two researchers. Two independent researchers extracted data on: study type; MSK pain population; type of TE used; definitions, parameters, measurement methods, and values of adherence.

Data sources: Seven electronic databases were searched from inception to December 2016.

Eligibility Criteria: Any study type featuring TE for adults with MSK pain and containing a definition of adherence, or a description of how adherence was measured.

Results: 459 studies were identified and 86 were included in the review. Most were prospective cohort studies and featured back and/or neck pain. Strengthening and stretching were the most common types of TE. A clearly identifiable definition of adherence was provided in $40 \%$ of the studies, with $12 \%$ using the same definition. Exercise frequency was the most commonly measured parameter of adherence, with self-report logs the most common measurement method. The most common value range used to determine satisfactory adherence was $80-99 \%$ of the recommended exercise dose.

Conclusion: No single definition of adherence to TE was apparent. We found no definition of adherence that specifically related to TE for MSK pain or described the dimensions of TE that should be measured. We recommend conceptualising adherence to TE for MSK pain from the perspective of all relevant stakeholders. 


\section{INTRODUCTION}

There is strong evidence for exercise therapy as a treatment option for musculoskeletal (MSK) pain in primary care, with medium or large effect sizes for pain, function and quality of life outcomes ${ }^{1}$. The effects of exercise compare favourably with other treatments including self-management, manual therapy, oral and topical pharmacological treatments, and surgery ${ }^{1}$. Therapeutic exercise (TE) is an effective and safe treatment for MSK pain ${ }^{2,3,4}$ and is recommended in many clinical guidelines $s^{5,6,7,8}$. However, the effectiveness of exercise is dependent upon the level of adherence to the recommended exercise protocol. A systematic review of 72 exercise treatments for low back pain (LBP) found that when adherence was encouraged to achieve a high dose of exercise, pain scores improved favourably compared to lower doses of exercise ${ }^{9}$. Higher exercise adherence can improve pain and physical function outcomes in patients with MSK pain and osteoarthritis $(O A)^{10,11}$. Adherence was identified by an expert consensus group as an important factor for determining outcomes from exercise in patients with knee or hip $\mathrm{OA}^{12}$. To improve the effectiveness of exercise for MSK pain, various strategies to enhance adherence have been investigated, such as goal setting and automated reminders but with inconsistent results ${ }^{13}$.

Three recent systematic reviews of measures of exercise and self-management methods for MSK pain $^{14,15,16}$, found that measures of exercise adherence currently used within randomised controlled trials are highly variable (including questionnaires, diaries and class registers), lack evidence of a robust or considered development process, and demonstrate inadequate psychometric properties for reliability and validity. There is no existing measure of adherence that is fit for purpose ${ }^{14,15,16}$. A valid and reliable measurement tool for adherence to TE for MSK pain is therefore required to enable interpretation of results from exercise trials and to robustly test the effectiveness of interventions intended to improve exercise adherence ${ }^{13-16}$.

To develop a measurement tool, the concept of interest must first be understood ${ }^{17}$ as the adequacy of an instrument will depend upon the conceptual framework from which it is developed ${ }^{18}$. However, exercise adherence is a multi-dimensional construct ${ }^{19}$, which is poorly defined ${ }^{16}$, with multiple synonyms used in the published literature to describe its meaning, such as: compliance, concordance, agreement, cooperation, partnership and therapeutic alliance ${ }^{20}$. These terms are regularly used interchangeably, although arguably were originally intended to convey different meanings. Compliance and adherence both refer to the patent-healthcare practitioner (HCP) interaction, but adherence is viewed as reflecting a less paternalistic relationship, with the patient as an active decision maker rather than passive recipient. Similarly, concordance is seen to better reflect the creation of a therapeutic alliance between the patient and $\mathrm{HCP}^{21}$. A common definition of 
adherence used in the healthcare literature is that created by Sackett and Haynes in $1976^{20,22}$, with the following modified iteration published in the World Health Organisation's (WHO) Adherence Project $(2003)^{23}:$ "the extent to which a person's behaviour - taking medication, following a diet, and/or executing lifestyle changes, corresponds with agreed recommendations from a healthcare provider". This definition was not specifically developed for MSK pain or TE, nor does it provide any guidance for operationalising the measurement of adherence in this context.

Therefore, to inform the development of a new measurement tool, this review aims to establish the dimensions of adherence to TE for MSK pain in adults reported in the published literature. The dimensions of adherence to TE among adolescents and children may be different to adults and warrants separate investigation. In this review, we use the term 'dimension' to refer to any single element that contributes towards the concept of adherence. In order to establish relevant dimensions of adherence, our objectives were: 1 . Identify specific definitions of adherence used in the context of TE for MSK pain; 2. Identify the parameters used to assess TE adherence (where a parameter is a measurable aspect of adherence); 3 . Identify the methods used to measure TE adherence; 4 . Identify values used to classify satisfactory TE adherence.

\section{METHODS}

\section{Search strategy}

To maximise the sensitivity of the search, three separate search strings were combined, including terms for MSK pain, therapeutic exercise, and adherence. The search strategy was adapted from a Cochrane review of interventions to improve adherence to exercise for chronic MSK pain in adults ${ }^{13}$. The adherence terms were limited to the title only to restrict the search to studies in which adherence was the primary focus. A pilot search was conducted to refine the focus of the strategy while maintaining sufficient sensitivity to identify key studies already known to the authors. The review was not registered a priori.

\section{Data sources}

Seven databases were searched from inception to December 2016 using the OvidSP and EBSCO interfaces: MEDLINE, AMED, EMBASE, PsychINFO, HMIC, CINAHL and SPORTDiscus. The Cochrane Database of Systematic Reviews was also searched and free text searching using Google Scholar was carried out. The reference lists of included studies were checked, and Web of Science was searched for papers that had cited included studies to identify further titles that may have met the inclusion criteria. Identified titles and abstracts, then subsequent full texts were screened by pairs of 
researchers $(\mathrm{DB}, \mathrm{AB}, \mathrm{MH}, \mathrm{JQ})$ independently, and any conflicts were arbitrated by an additional researcher (NF).

\section{Studies were eligible if they featured all of the following:}

1. A definition of exercise adherence - either an explicit definition such as a quote or citation, or a description of how exercise adherence was or should be measured.

2. Adults with MSK pain - including back, neck, hip, knee, ankle, foot, shoulder, elbow, wrist or hand pain, MSK conditions with wider systemic effects such as fibromyalgia and rheumatoid arthritis, post-surgical pain patients where the surgery was for a MSK condition, individuals with a non-specific diagnosis of MSK pain, and those with a specific diagnosis (e.g. OA or adhesive capsulitis) with or without supporting imaging or other diagnostic test results.

3. Therapeutic exercise - defined as any form of supervised or unsupervised exercise or physical activity specifically provided to patients to treat their MSK pain condition. Any type of exercise was included, such as strengthening, stretching, aerobic or mixed, and exercise delivered by an HCP, trained lay representative, fitness instructor, or delivered as part of a multi-disciplinary package of care. All study types were eligible, not just those where therapeutic exercise was being assessed as an intervention.

\section{Studies were excluded if they:}

1. Were not published in English.

2. Comprised conference proceedings where only the abstract has been published.

3. Involved exercise for a non-MSK pain condition, such as cancer, falls prevention, stroke or cardiac rehabilitation.

4. Included participants under 18 years.

5. Included participants with no MSK pain.

\section{Data extraction}

Data were extracted independently by two researchers using customised forms. Extracted data were compared for inconsistencies and any corrections made following discussion by the researchers. A third researcher was consulted if needed. We extracted: details of the study (design, country, setting), participant details (age, sample size, population, inclusion/exclusion criteria, MSK pain condition), the TE intervention (type, dosage, adherence measure time-point, exercise prescriber, supervised or unsupervised exercise), definitions of adherence (quotes or citations were extracted verbatim), the parameters of exercise adherence assessed (e.g. number of repetitions, attendances or intensity of exercise), methods used for measuring adherence (e.g. class register or self-report diary), and quantification or values assigned to adherence (e.g. number of exercises that 
should be completed to be classed as adherent). Systematic reviews and protocols were included if they met the above criteria as it was possible they could include information about how adherence is defined. However, only data on the definition of adherence was extracted from systematic reviews to avoid the over-representation of studies that may have been included in a systematic review as primary research in this review.

\section{Analysis}

Data from included studies were summarised in tables. Terms used for describing parameters and measurement methods were standardised and frequency and percentage counts applied. Values for quantification of adherence were standardised by converting to percentages for ease of comparison and grouped according to commonly observed ranges. We did not formally assess the risk of bias since the methodological quality of included studies would not have influenced the utility or relevance of the data that were extracted for the purposes of this systematic review.

\section{RESULTS}

We identified 459 references, which reduced to 199 following screening of titles and abstracts. Finally, 86 studies met the inclusion criteria and were included in the review (Figure 1).

\section{Characteristics of included studies}

Included studies were published between 1976 and 2016 and originated from 20 different countries (Table 1). Sixteen different study types were included and hospital outpatients was the most common study setting $(n=31)$. Seventeen different categories of MSK pain conditions featured, most commonly back and/or neck pain $(n=30)$. The type of TE could be broadly grouped as: strengthening, stretching, aerobic, postural or mind-body exercise ${ }^{24}$ (e.g. yoga), with strengthening $(n=43)$ and stretching $(n=35)$ being the most common. The time-point at which exercise adherence was measured in the studies varied from 1 week to 5 years.

\section{Definitions of exercise adherence}

Adherence data findings extracted from included studies are shown in appendix 2 . Sixty per cent of studies $(n=52)$ did not provide a clearly specified definition of adherence. Most studies defined adherence by describing the parameters by which it had been assessed ( $n=73,85 \%$ of 86 ).

Thirty-four studies (40\%) provided at least one clearly specified definition, the most common of which are shown in Table 2. Five studies provided definitions that were the authors' own or were uncited. Frost et al. $(2016)^{25}$ provided their own definition in addition to that of the WHO. Their 
definition, "the extent to which individuals undertake prescribed behaviour accurately and at the agreed frequency, intensity and duration" was based on the four parameters of adherence they believed characterised rehabilitation prescriptions, namely: frequency, duration, intensity and accuracy.

Table 2. Definitions of adherence used in studies

\section{Definition and source}

"The extent to which a person's behaviour - taking medications, following a diet, and/or executing lifestyle changes, corresponds with agreed recommendations from a healthcare provider". (WHO 2003)

"The extent to which the patient's behaviour (in terms or taking medications, following diets or executing other lifestyle changes) coincides with the clinical prescription". (Sacket and Haynes 1976)

"Adherence is defined by the active cooperation and the attitude of the patient during the therapy session and during home exercise execution". "The term adherence refers to the extent to which patients follow the instructions of their healthcare providers". "Adherence is defined as the extent to which the patient undertakes the clinic-based and home-based prescribed components of the physiotherapy programme". (all attributed to Meichenbaum and Turk 1987)

"Where adherence implies active voluntary involvement in the planning and implementation of the treatment and is defined as the extent to which the patient undertakes the clinic-based and home-based prescribed components of the physiotherapy programme." "Adherence, which denotes a more contemporary approach to decision making in which the client or patient is an active and equal partner with the health professional." (both attributed to Carr 2001)

\section{Studies citing definition}

Beinart et al. (2013)

Ezzar et al. (2014)

Hall et al. (2014)

Holden et al. (2014)

Frost et al. (2016)

Jack et al. (2010)

Jordan et al. (2010)

Pisters et al. (2010)

Thompson et al. (2016)

Van Koppen et al. (2016)

Alexandre et al. (2002)

Brus et al. (1997)

Brus et al. (1998)

Ferguson and Bole (1979)

Hammer et al. (2007)

Medina-Mirapeix et al. (2009)

Robinson et al. (2004)

Schneider et al. (1998)

Wig et al. (2004)

Hugli et al. (2014)

Huyser et al. (1997)

Mannion et al. (2009)

Mannion et al. (2009)

Marks and Allegrante (2005)

\section{Parameters used to assess exercise adherence}

Frequency of exercise completion was the most commonly used parameter to assess adherence $(n=47,55 \%)$ (Table 3). Frequency was measured in different ways, including: exercise repetitions, 
sets, or blocks of exercise time, and over different time-frames (e.g. exercises per day, week or month).

Behavioural parameters were measured to assess adherence in 16 studies (19\% of 86 ). These included HCP assessed elements such as 'following guidance' and 'receptivity to change', as featured in the Sports Injury Rehabilitation Scale (SIRAS) ${ }^{19}$, and self-reported elements such as 'barriers to exercise'. Session attendance, which required the patient to be present at a supervised exercise session, was assessed in 22 (26\%) studies. This was slightly different to 'session completion' assessed in $11(13 \%)$ studies, which was a self-reported or HCP observed completion of recommended exercises during an exercise session.

Exercise exertion or intensity was assessed in 13 studies (15\%), subjectively in eight (9\%) and objectively in five (6\%). Seven studies (8\%) assessed a parameter reflecting the quality of the patients' replication of the recommended exercises, which was determined via HCP observation.

Table 3. Number of studies describing parameters used to assess TE adherence

\section{Parameter of TE adherence measured Number of studies using parameter (\%)}

\begin{tabular}{lc}
\hline Exercise frequency & $47(55 \%)$ \\
\hline Session attendance & $22(26 \%)$ \\
\hline Behavioural component & $16(19 \%)$ \\
\hline Exercise time & $15(17 \%)$ \\
\hline Sessions completed & $11(13 \%)$ \\
\hline Exercise exertion (subjective measure) & $8(9 \%)$ \\
\hline Exercise replication & $7(8 \%)$ \\
\hline Exercise intensity (objective measure) & $5(6 \%)$
\end{tabular}

(NB. Some studies described more than one parameter, hence totals do not sum to $100 \%$ )

\section{Methods used to measure adherence}

Of the 86 included studies, 74 (86\%) described at least one method of measuring adherence. The most common measurement methods were self-report exercise logs $(n=44,51 \%)$, registers of 
attendance $(n=18,21 \%)$ and an existing measurement scale $(n=15,17 \%)$. Thirty-three studies (38\%) used more than one type of measurement method. Six studies used an objective measure of exercise adherence (e.g. pedometer) (Table 4). Within the category of 'existing measurement scales', 12 different measures of exercise adherence were identified. These included the SIRAS ${ }^{19}$, General Adherence Scale $(\mathrm{GAS})^{26}$ and Adherence to Physiotherapists Recommendation Scale (APRS) ${ }^{27}$.

Table 4. Number of studies describing a method of measuring TE adherence

Measurement method Number of studies using method (\%)

\begin{tabular}{lc}
\hline Self-report exercise log & $44(51 \%)$ \\
\hline Class register & $18(21 \%)$ \\
\hline Existing measurement scale & $15(17 \%)$ \\
\hline Healthcare practitioner observation & $11(13 \%)$ \\
\hline Self-developed questions & $12(14 \%)$ \\
\hline Objective measure & $6(7 \%)$ \\
\hline Interview & $6(7 \%)$ \\
\hline
\end{tabular}

(NB. Some studies described more than one method, hence totals do not sum to $100 \%$ ).

\section{Values for adherence}

Half of the studies $(n=44,51 \%)$ did not provide information about what they considered to be a satisfactory value for TE adherence. Forty-two of the studies (49\%) described values indicating 'satisfactory' TE adherence (Table 4). These were grouped into four ranges as shown in Table 5 . The most common range of values for satisfactory adherence was between $80-99 \%$ completion of the prescribed exercise/s. Six studies (7\%) provided values specifically describing 'low adherence'. These varied between $0-79 \%$ completion of the recommended exercises. It was unclear in many of the studies whether cut-off points for satisfactory adherence were determined a priori or post hoc, as they were not described in the methods. Where cut-off points were stated, no references to required therapeutic dosages or other guidelines were mentioned. Some studies used the distribution of participants' adherence data to assign a value of satisfactory adherence. For example, Granlund et al. $(1998)^{28}$ used the median value of participants' adherence results to 
dichotomise them into adherent or non-adherent groups, whereas Van Gool et al. (2005) ${ }^{29}$ ordered the participants according to their adherence results, then divided them into three equally sized groups described as 'low', 'intermediate' or 'high' adherence.

Table 5. Number of studies stating values for satisfactory TE adherence

\begin{tabular}{|cc}
\hline Value for satisfactory adherence & Number of studies using this level \\
\hline $100 \%$ of recommended dose & $8 \%)$ \\
\hline $80-99 \%$ of recommended dose & $15(17 \%)$ \\
\hline $60-79 \%$ of recommended dose & $9(10 \%)$ \\
\hline $14-59 \%$ of recommended dose & $10(12 \%)$ \\
\hline No value given & $44(51 \%)$ \\
\hline
\end{tabular}

\section{DISCUSSION}

We aimed to establish the dimensions of adherence to TE for MSK pain, in other words, all information reported in relevant published literature that contributes towards the concept or meaning of adherence. This was achieved by identifying: specific definitions of TE adherence; the parameters used to assess TE adherence; the methods used to measure TE adherence and values for satisfactory adherence. We included studies from a wide range of countries featuring various methodologies, settings, MSK pain conditions, and TE interventions. Most commonly, study authors did not state a definition of TE adherence, even when this was a focus of their study. Where a definition was stated, most originated from the work of Sackett and Haynes (1976) ${ }^{22}$.

Exercise frequency was the most common parameter by which TE adherence was assessed, although it was commonly combined with other parameters. A variety of methods of TE adherence measurement were reported. A self-report exercise log was the most frequently used method, although the structure and implementation of these methods varied between studies. Most studies did not define what was considered a satisfactory level of adherence, however $80-99 \%$ completion of the recommended exercises was most frequently used as a surrogate of satisfactory adherence. 


\section{Definitions of adherence}

Most studies did not state a definition of adherence. This may be because a definition of adherence that is explicitly related to adherence to TE does not exist. Alternatively, it might be that the meaning of adherence is so clear, having undergone so little development between the 1976 version by Sackett and Haynes to the 2003 version from the WHO, that there is an assumption that the term is tacitly understood among research and clinical communities. However, the lack of an agreed communicable definition of adherence is a problem, because without a clear picture of what adherence is, HCPs and patients cannot work together to achieve adherence as a shared goal, nor measure or monitor its variability.

The original definition by Sackett and Haynes $(1976)^{22}$ used the term 'compliance' not 'adherence', and concerns therapeutic regimens generally, not specifically MSK pain or TE. While the more recent WHO iteration of this definition (2003) ${ }^{23}$ uses the term 'adherence', to better reflect the autonomy of the patient and his/her role as an 'active partner' in the treatment process, it was developed by groups of experts focussing on adherence issues relating to chronic health conditions other than MSK pain (including hypertension, epilepsy, HIV and tuberculosis). Consequently, pharmacological intervention was the main treatment of concern for both these definitions, but it is not clear whether adherence to TE can be defined in the same way. These possible differences may explain why the WHO definition (2003) $)^{23}$ has not been universally applied to TE for MSK pain. The WHO definition lacks specificity, providing no indication of the parameters of treatment adherence that should be assessed, how they should be measured, or what levels of adherence should be expected. This ambiguity means definitions can be interpreted differently, as shown by this review, where several interpretations of a single definition were associated with authors using different parameters, measurement methods and cut-offs.

It has been proposed that the term compliance should be used in efficacy trials where the intervention has been determined according to the desired outcome and target population, but not necessarily the individual patient or recipient ${ }^{30}$. Adherence on the other hand, is proposed to reflect the effectiveness of TE in trials or real-life contexts ${ }^{30}$. Adherence considers the individual's role in interpreting the appropriateness of the advised intervention and the influences upon their behaviour and motivation, such as the environment, society, previous experience, knowledge, symptoms, and resources. Adherence is a more complex measurement than simply comparing to a reference standard intervention dose $\mathrm{e}^{30}$. Until the message we are trying to convey with these terms is clarified by all those to whom it pertains, including patients, the uptake of existing definitions may remain inconsistent in MSK pain and TE literature. 
The definition of adherence provided by Frost et al. $(2016)^{25}$ is specific to TE for MSK pain and identified the parameters of TE adherence that should be measured: frequency, duration, intensity, and accuracy. However, its development was based on stroke rehabilitation ${ }^{31}$, public health ${ }^{32}$, falls prevention $^{33}$ and the use of exercise sheets in physiotherapy, ${ }^{34}$ not literature focussing specifically on TE prescription for MSK pain. Therefore, it is possible that the definition does not reflect the perceptions of patients with MSK pain or HCPs responsible for prescribing TE. While Frost et al. $(2016)^{25}$ offer the first operational definition of TE adherence, its four parameters are different to those identified as most commonly used in this review. Therefore, the concept of adherence to TE for MSK pain may require further refinement before the development of a new measure of adherence.

\section{Parameters of adherence}

We identified 8 categories of TE adherence parameters (measurable aspects of adherence). The most common was exercise frequency, a finding consistent with other reviews, suggesting it is a relevant dimension of TE adherence, or simply an easy parameter to measure. However, even exercise frequency was assessed in various ways (e.g. repetitions or blocks of time, per day or week), suggesting it is more complex than a simple representation of the total TE undertaken.

The accuracy with which patients replicated their exercises, or the quality of their exercise performance was included as a parameter in 7 studies. The scant attention paid to accuracy or quality of performance may be because it is not an important dimension of TE adherence, or that it is too challenging to incorporate into research, despite being a common parameter of adherence to assess in clinical practice ${ }^{15}$. HCP observation has been recommended in the assessment of TE adherence $\mathrm{e}^{35}$ and is a feature of one of the more commonly used measures: the SIRAS ${ }^{36}$. For research purposes, the constraint of requiring an observer and its associated cost implications may be why these parameters have not been regularly assessed. While technology may be able to objectively measure certain parameters of $\mathrm{TE}^{37}$, the expense of such equipment, the expertise required to operate it and the number of sensors required to measure more complex TE interventions, may limit its implementation ${ }^{38}$. The acceptability of the equipment by patients may also limit its effectiveness as a measurement tool, as patients may not adhere to using the measurement device, despite adhering to the TE intervention. Natural adherence behaviour may also be affected by overt monitoring, such as wearing a measurement device, although similar changes may also be associated with subjective measurement methods.

Behavioural components featured in 16 studies, two of which, used self-reported barriers to exercise completion as a parameter of adherence ${ }^{39,40}$. This is an uncommon approach, as barriers are 
normally seen as modifiers of adherence levels. However, poor treatment adherence has been associated with a greater perceived number of barriers ${ }^{41}$ indicating a potential role for barriers in the assessment of adherence. Some studies identified single parameters of TE adherence, whereas other studies identified combinations of parameters (e.g. exercise frequency and session attendance), without justification. These findings suggest there are multiple relevant parameters of adherence to $\mathrm{TE}$, but no consensus as to their importance or relevance to a specific context. This may relate to non-specific definitions such as the WHO's focus on pharmacological interventions, whereas TE prescription may potentially have more parameters contributing to adherence, such as: frequency, intensity, time, accuracy or a behavioural component, which require different methods for their measurement.

\section{Methods of measuring adherence}

Multiple methods of measuring exercise adherence were identified, including self-report exercise logs, class registers and existing measurement scales, mirroring the findings from recent systematic reviews $^{14,15,16}$. However, different measurement methods were used across studies for the same adherence parameter. For example, the parameter of session attendance was measured using a class register, interview and self-report log. Such variation is understandable given the multiple parameters of adherence and the non-specific guidance provided by the WHO's adherence report ${ }^{23}$, which suggests, "a multi-method approach combining self-reporting and objective measures". However, the lack of a single valid and reliable measurement tool of TE adherence means that the relative effectiveness of interventions is difficult to compare across studies ${ }^{13,14,15,16}$.

There was large variability in time points at which adherence was measured, varying from 1 week to 5 years. As adherence is likely to change over time ${ }^{13}$, the point at which adherence is measured could substantially influence the rates of adherence observed. To improve the comparability of data, it may be useful to establish adherence measurement timescales (e.g. short, medium and longterm). There should also be improved clarity in the reporting of adherence measurement timing (e.g. whether measurements took place daily, weekly or monthly), and for studies where there are multiple deliveries of the TE intervention (e.g. weekly exercise classes), the time interval between the TE delivery and the adherence measurement.

\section{Values of adherence}

Fewer than half of included studies (49\%) defined satisfactory adherence. Two approaches were used, either a pre-determined cut-off or a distribution method. Distribution-based methods result in the adherence of an individual being judged relative to the performance of other study participants, rather than any pre-determined target level of adherence. This explains why satisfactory adherence 
levels ranged from $14-100 \%$ completion of prescribed exercises in this review. Only one study, Hammer et al. (2007) $)^{42}$ used pre-determined adherence categories justified from the literature. ${ }^{43}$ However, the original source provides no explanation of how these categories were derived. In our review, satisfactory adherence was most commonly valued between $80-99 \%$ completion of the prescribed exercises $(n=15,17 \%)$. Furthermore, several studies described low adherence, providing ranges of prescribed exercise completion between 0 and $79 \%$. This may suggest that $80 \%$ could be a reasonable threshold for satisfactory adherence.

If specific TE doses are unknown, it may be inappropriate and unhelpful to set arbitrary adherence cut-off points for TE interventions for MSK pain. A systematic review and meta-analysis of medication adherence showed that good adherence to placebo medication was associated with lower mortality, a so called 'healthy adherer' effect ${ }^{44}$. Therefore, it may be beneficial for a patient to identify as adherent, even in the absence of a known therapeutic dosage. Identifying an MSK patient as non-adherent when the therapeutic dosage of TE is unknown, may deny the patient the benefits of the healthy adherer effect.

\section{Research and clinical Implications}

There is a need to focus on establishing a clear definition of adherence specifically relating to TE for MSK pain, and the best way to measure adherence. Patients must be involved as active, collaborative partners in future research so that the resulting construct of exercise adherence incorporates their views ${ }^{45}$. In the absence of a valid, reliable and acceptable measure of TE adherence for MSK pain, the interpretation of results from trials investigating TE interventions or methods for improving TE adherence may be questioned. This underscores the importance of agreeing upon a satisfactory measure/s. From the clinician's perspective, if he or she cannot be sure of how to accurately define and measure adherence, it may be difficult to determine whether a patient's lack of progress despite treatment is due to inadequate engagement or ineffective treatment.

\section{Limitations}

We adopted an inclusive approach using a modified versions of an established Cochrane search strategy to include all study types and MSK pain conditions, however, it is possible that some studies relating to adherence to TE for MSK conditions may have been missed. To minimise error and bias, two independent reviewers assessed both title and abstract and full text papers for inclusion and data extraction. The search terms were limited to title, or title and abstract. Restricting the search in this way, ensured that the included studies were specifically focused on adherence in the context of TE for MSK pain and therefore more likely to be appropriate to this review. It is possible that some 
relevant studies may have been missed that did not feature adherence in their title. However, different search strategies were piloted to achieve results with a focus on adherence while maintaining acceptable sensitivity.

Since a definition of adherence was not provided in the majority of included studies despite their title featuring a term for adherence, it is unlikely that articles not featuring an adherence term in their title would discuss the concept in any detail. Equally, the majority of definitions used were from the same source, and it is unlikely that this would change if more articles were reviewed. Six studies were excluded as they were not published in English. Consequently, our review is at risk of language bias, and the results may under-represent studies that are not published in English. The countries of origin of the excluded studies were still represented in the final 86 included studies. No low-income countries were studied. Therefore, our findings may only be generalisable to high income countries and their cultures.

\section{CONCLUSION}

Most studies did not state a definition of adherence. Definitions most commonly used in the context of TE for MSK pain were not developed specifically for TE or MSK pain, and did not describe the context specific dimensions of this concept. The variability of the parameters of adherence assessed, the wide variety of measurement methods used, and the seemingly arbitrary nature of determining values for satisfactory adherence, lack sufficient consistency and detail as to inform a definition of adherence or the required content of a suitable measure. 


\section{REFERENCES}

1. Babatunde OO, Jordan JL, Van der Windt DA, Hil JC, Foster NE, Protheroe J (2017) Effective treatment options for musculoskeletal pain in primary care: A systematic overview of $\begin{array}{lllll}\text { current PloS ONE } & \text { 1296): } 0178621 .\end{array}$ https://doi.org/10.1371/journal.pone.0178621

2. Rainville J, Hartigan C, Martinez E, Limke J, Jouve C, Finno M 2004 Exercise as a treatment for chronic low back pain. The Spine Journal 4:106-115

3. UK BEAM Trial Team 2004 United Kingdom back pain exercise and manipulation (UK BEAM) randomised trial: cost effectiveness pf physical treatments for back pain in primary care. BMJ, doi:10.1136/bmj.38282.607859.AE (published 29 November 2004)

4. Tan SS, Teirlinck CH, Dekker J, Goossens LMA, Bohnen AM, Verhaar JAN, van Es PP, Koes BW, Bierma-Zeinstra SMA, Luijsterburg PAJ, Koopmanschap MA 2016 Cost-utility of exercise therapy in patients with hip osteoarthritis in primary care. Osteoarthritis and cartilage 24(4):581-588

5. British Pain Society (BPS) 2013. Guidelines for Pain Management Programmes for adults. https://www.britishpainsociety.org/static/uploads/resources/files/pmp2013_main_FINAL_v 6.pdf. Accessed $5^{\text {th }}$ July 2017.

6. National Institute for Health and Care Excellence (NICE) 2009. Low back pain: early management of persistent non-specific low back pain. https://www.nice.org.uk/guidance/cg88?unlid=5626398422015113014535. Accessed 18th July 2014.

7. National Institute for Health and Care Excellence (NICE) 2014. Osteoarthritis: care and management in adults.

https://www.nice.org.uk/guidance/cg177?unlid=38189754201622015349. Accessed $5^{\text {th }}$ July 2017.

8. New Zealand Guidelines Group (NZGG). The diagnosis and management of soft tissue shoulder injuries and related disorders. 2003. New Zealand. http://www.acc.co.nz/PRD_EXT_CSMP/groups/external_communications/documents/guide /wcm001684.pdf. Accessed $5^{\text {th }}$ July 2017.

9. Hayden JA, van Tulder MW, Tomlinson G (2005) Systematic review: Strategies for using exercise therapy to improve outcomes in chronic low back pain. Ann Intern Med 142: 776785.

10. Jakobsen MD, Sundstrup E, Brandt M, Anderson LL (2016) Factors affecting pain relied in response to physical exercise interventions among healthcare workers. Scand J Med Sci Sports doi:10.1111/sms.12802

11. van Gool CH, Penninx BW, Kempen GI, Rejeski WJ, Miller GD, Van Eijk JT, Pahor M, Messier SP (2005) Effects of exercise adherence on physical function amoung overweight adults with knee osteoarthritis. Arthritis Rheum 53(1): 24-32

12. Roddy E, Zhang W, Doherty M, Arden KM, Barlow J, Birrell F, Carr A, Chakravarty K, Dickson J, Hay E, Hosie G, Hurley M, Jordan KM, McCarthy C, McMurdo M, Mockett S, O'Reilly S, Peat G, Pendleton A, Richards S (2005) Evidence-based recommendations for the role of exercise in the management of osteoarthritis of the hip or knee-the MOVE consensus. Rheumatology 44:67-73.

13. Jordan JL, Holden MA, Mason EE and Foster NE (2010) Interventions to improve adherence to exercise for chronic musculoskeletal pain in adults. Cochrane Database of Systematic Reviews, pp. (1)-2010.

14. Bollen JC, Dean SG, Siegert RJ, Howe TE, Goodwin VA (2014) A systematic review of measures of self-reported adherence to unsupervised home-based rehabilitation exercises programmes, and their psychometric properties. BMJ Open 4:e005044 doi:10.1136/bmjopen-2014-005044 
15. McClean S, Holden M, Potia T, Gee M, Mallett R, Bhanbhro S, Parsons H, Haywood K (2017) Quality and acceptability of measures of exercise adherence in musculoskeletal settings: a systematic review. Rheumatology 56(3): 426-438

16. Hall AM, Kamper SJ, Nernon M, Hughes K, Kelly G, Lonsdale C, Hurley DA, Ostelo R (2015) Measurement tools for adherence to non-pharmacological self-management treatment for chronic musculoskeletal conditions: a systematic review. Archives of Physical Medicine and Rehabilitation 96(3):552-556

17. de Vet HCW, Terwee CB, Mokkink LB, Knol DL (2011) Measurement in Medicine. Cambridge University Press, NY.

18. U.S Department of Health and Humand Services Food and Drug Administration (2009) Guidance for industry patient-reported outcome measures: use in medical product development to support labelling claims. Accessed May 2017 https://www.fda.gov/downloads/drugs/guidances/ucm193282.pdf

19. Kolt GS, Brewer BW, Pizzari T, Schoo AMM, Garrett N (2007). The sport injury rehabilitation scale: a reliable scale for use in clinical physiotherapy. Physiotherapy 93:17-22

20. Bissonette JM (2008) Adherence: a concept analysis. Journal of Advanced Nursing 63(6): 634-643

21. Horne R, Weinman J, Barber N, Elliot R, Morgan M (2005) Concordance, adherence and compliance in medicine taking. Report for the National Co-ordinating Centre for NHS Service Delivery and Organisation R \& D (NCCSDO)

22. Sackett DL, Haynes RB (1976) Compliance with Therapeutic Regimens. The John Hopkins University Press, Baltimore, MD.

23. World Health Report (2003) Adherence to long term therapies, evidence for action. Geneva, World Health Organisation, 2003. ISBN 924545992110 pages, order no 1150526.

24. Brosseau L, Taki J, Desjardins B, Thevenot O, Fransen M, Wells GA, Imoto AM, Toupin-April K, Westby M, Gallardo ICÁ, Gifford W, Laferrière L, Rahman P, Loew L, Angelis G, Cavallo S, Shallwani SM, Aburub A, Bennell KL, Van der Esch M, Simic M, McConnell S, Harmer A, Kenny GP, Paterson G, Regnaux JP, Lefevre-Colau MM, McLean L (2017) The Ottawa panel clinical practice guidelines for the management of knee osteoarthritis. Part one: introduction, and mind-body exercise programs. Clin Rehabil 31(5): 582-595

25. Frost R, Levati S, McClurg D, Brady M, Williams B (2016) What adherence measures should be used in trials of home-based rehabilitation interventions? A systematic review of the validity, reliability and acceptability of measures. Archives of Physical Medicine and Rehabilitation DOI 10.1016/j.apmr.2016.08.482 article in press

26. Dobkin PL, Ionescu-Ittu R, Abrahamowicz M, Baron M, Bernatsky S, Sita A (2008) Predictors of adherence to an integrated multimodal program for fibromyalgia. J Rheumatol 35: 22552264

27. Lonsdale C, Hall AM, Williams GC, McDonough SM, Ntoumanis N, Murray A, Hurley D (2012) Communication style and exercise compliance in physiotherapy (CONNECT). A cluster randomized controlled trial to test a theory-based intervention to increase chronic back pain patients' adherence to physiotherapists' recommendations: study rationale, design, and methods. BMC Musculoskeletal Disorders 12: 104

28. Granlund B, Brulin C, Johansson H, Sojka P (1998) Can motivational factors predict adherence to an exercise program for subjects with low back pain? Scandinavian Journal of Behavioural Therapy 27(2):81-96

29. Van Gool CH, Penninx BW, Kempen GI, Rejeski WJ, Miller GD, Van Eijk JT, Pahor M, Messier SP (2005) Effects of exercise adherence on physical function amoung overweight adults with knee osteoarthritis. Arthritis Rheum 53(1): 24-32

30. McKay CD 2016 Compliance versus adherence in sports injury prevention: Why definition matters. Br J Sports Med 50:382-383 
31. Page SJ, Schmid A, Harris JE (2012) Optimising terminology for stroke rehabilitation: recommendations from the American Congress of Rehabilitation Medicine Stroke Movement Interventions Subcommittee. Arch. Phys. Rehabil. 93:1395-9.

32. Pollock M, (1988) Prescribing Exercises for Fitness Adherence. In: Exercise Adherence: Its Impact on Public Health. Champaign: Human Kinetics p259-77.

33. Simek EM, McPhate L, Haines TP (2012) Adherence to and efficacy of home exercise programs to prevent falls; A systematic review and meta-analyisis of the impact of exercise progam characteristics. Prev. Med. (Baltim) 55:262-75.

34. Smith J, Lewis J, Prichard D. (2005) Physiotherapy exercise programmes: Are instructional exercise sheets effective? Physiother. Theory Pract 21:93-102.

35. Bassett SF (2003) The assessment of patient adherence to physiotherapy rehabilitation. NZ Journal of Physiotherapy 31(2): 60-66.

36. Brewer BW, Van Raalte JL, Petitpas AJ, Sklar JH, Pohlman MH, Krushell, RJ, Ditmar TD, Daly JM, Weinstock J (2000) Preliminary psychometric evaluation of a measure of adherence to clinic-based sport injury rehabilitation. Physical Therapy in Sport 1: 68-74.

37. Giggins OM, Sweeney KT, Caulfield B (2014) Rehabilitation exercise assessment using inertial sensors: a cross sectional analytical study. Journal of Neuroengineering and Rehabilitation 11:158

38. Yang C, Hsu L (2010) A review of accelerometry-based wearable motion detecors for physical activity monitoring. Sensors 10:7772-88

39. Van Koppen LH, Zandwijk PJJ, Van Mameren HV, Mesters I, Winkens B, De Bie RA (2016) Patients' adherence to a walking programme for non-specific low back pain. European Journal of Physiotherapy 18(2):1-16

40. Zandwijk PJJ, Van Koppen LH, Van Mameren H, Mesters I, Winkens B, De Bie R (2015) The accuracy of self-reported adherence to an activity advice. European Journal of Physiotherapy 17(4):183-191

41. Jack K, Mclean SM, Moffett JK, Gardiner E (2010) Barriers to treatment adherence in physiotherapy outpatient clinics: A systematic review. Manual Therapy 15:220-228

42. Hammer C, Degerfeldt L, Denison E (2007) Mechanical diagnosis and therapy in back pain: compliance and social cognitive theory. Advances in Physiotherapy 9:190-197

43. Chen C-Y, Strecker Neufeld P, Feely CA, Sugg Skinner C. Factors influencing compliance with home exercise programs among patients with upper-extremity impairment. Am J Occup Ther. 1999; 53:171-80.

44. Simpson SH, Eurich DT, Majumdar SR, Padwal RS, Tsuyuki RT, Varney J, Johnson JA (2006) A meta-analysis of the association between adherence to drug therapy and mortality. BMJ 333:15-21.

45. Staniszewska S, Haywood KL, Brett J, Tutton L. (2012) Patient and public involvement in patient-reported outcome measures: evolution not revolution. Patient 5:79-87.

\section{SUMMARY}

- Patients' adherence to therapeutic exercise prescribed for MSK pain is important for outcomes.

- The most commonly used definition of adherence was not developed specifically for MSK pain and lacks detail on the important dimensions or cut-point from which to determine satisfactory adherence.

- The meaning of adherence in the context of therapeutic exercise for MSK pain is unclear and should be conceptualised by relevant stakeholders. 
